\title{
ERR $\gamma$ is downregulated in injured motor neuron subpopulations following brachial plexus root avulsion
}

\author{
GUANGYIN YU ${ }^{1 *}$, PRINCE LAST MUDENDA ZILUNDU ${ }^{1 *}$, LINLIN LIU $^{1 *}$, \\ KE ZHONG ${ }^{1}$, YING TANG ${ }^{1}$, ZEMIN LING $^{1}$ and LI-HUA ZHOU ${ }^{2}$ \\ ${ }^{1}$ Department of Anatomy, Zhongshan School of Medicine; ${ }^{2}$ Department of Anatomy, School of Medicine, \\ Sun Yat-sen University, Guangzhou, Guangdong 510080, P.R. China
}

Received March 2, 2019; Accepted September 19, 2019

DOI: $10.3892 /$ etm.2019.8209

\begin{abstract}
Estrogen-related receptor $\gamma(E R R \gamma)$ is a member of a small group of orphan nuclear receptor transcription factors that have been implicated in several physiological and pathological processes, including placental development, regulation of metabolic genes or disease. The pattern of expression of ERR $\gamma$, its role in neuronal injury and its co-localization with other transcription factors in the spinal cord of rats with brachial plexus injury has not been determined. The expression profile of ERR $\gamma$ and its co-localization with RNA binding protein fox-1 homolog $3(\mathrm{NeuN})$ or cyclic AMP-dependent transcription factor 3 (ATF-3) in the motor neurons of rats that underwent brachial plexus root avulsion were assessed using western blot analysis, immunohistochemistry and immunofluorescence. Fluorogold (FG) was used to mark neurons whose axons were severed. ATF-3 was expressed in the nuclei of motor neurons whose axons were severed by root avulsion. On day 3 post-avulsion, FG and ATF-3 were all co-localized in the injured motor neurons. The level of ERR $\gamma$ protein in the ipsilateral half of injured spinal cords was significantly decreased compared with that in the contralateral half on days 3, 14 and 28 post-avulsion (all $\mathrm{P}<0.05$ ). The numbers of ERR $\gamma$-positive motor neurons $\left(E R R \gamma^{\text {on }}\right)$ were also notably decreased in the ipsilateral side compared with that in the contralateral side on days 14 and 28 post-avulsion, implying that the expression occurred in $\alpha$ motor neurons that were progressively being lost, a phenomenon that was expected post-brachial plexus avulsion. Almost all large and small ERR $\gamma$-positive motor neurons were also NeuN-positive $\left(\mathrm{NeuN}^{\mathrm{on}}\right)$. However, a few of these were $\mathrm{ERR} \gamma^{\text {on }} / \mathrm{NeuN}^{\text {off }}$ (no NeuN signal). Therefore, these
\end{abstract}

Correspondence to: Professor Li-Hua Zhou, Department of Anatomy, School of Medicine, Sun Yat-sen University, 74 Zhongshan Road 2, Guangzhou, Guangdong 510080, P.R. China

E-mail: zhoulih@mail.sysu.edu.cn

*Contributed equally

Key words: estrogen-related receptor $\gamma$, motor neuron injury, brachial plexus root avulsion, $\alpha$ motor neurons, $\gamma$ motor neurons results suggested that ERR $\gamma$ is a non-specific marker of $\gamma$ motor neurons in rats, and therefore, this specific transcriptional program cannot be used to define functionally distinct motor neuron sub-populations. However, its downregulation on the injured side suggests that it is an important component of the response to injury in motor neurons.

\section{Introduction}

Brachial plexus root avulsion (BPRA), as a primary injury, is one of the most serious axonal injuries that may lead to multiple pathophysiological processes in the spinal cord $(1,2)$. These secondary processes often involve an altered expression of specific genes and proteins associated with inflammation, apoptosis, mitochondrial oxidative phosphorylation and oxidative stress, which, in turn, contribute to the apoptotic death of the affected motor neurons with subsequent permanent paralysis of the ipsilateral upper limb muscles (2).

The spinal cord ventral horn is where the somatic motor neuron subtypes, namely $\gamma, \beta$ and $\alpha$ motor neurons, are located. These subtypes are responsible for functional movements that are lost following a brachial plexus/spinal cord injury (3). $\beta$ motor neurons are the smallest and less abundant of the somatic motor neuron subtypes. The molecular and electrical properties of $\beta$ motor neurons are poorly characterized and largely remain unidentified $(3,4)$. $\gamma$ motor neurons contribute $\sim 30 \%$ to the motor neuron pool and have smaller cell bodies compared with the largest motor neurons, $\alpha$ motor neurons (1-4). Functionally, $\gamma$ motor neurons innervate the intrafusal muscle fibers, which modulate the sensitivity of muscle spindles to stretch (3-5). $\alpha$ motor neurons are the predominant cell type in the motor neuron pool. They innervate force-generating extrafusal muscle fibers at neuromuscular junctions. Notably, in mice, the muscle fiber targets of $\gamma$ and $\alpha$ motor neurons can be distinguished by estrogen-related receptor (ERR) transcription factor expression (5). It is unknown if the same is true for rats, which represent an important experimental animal model for brachial plexus injuries.

ERRs are a small group of orphan nuclear receptor transcription factors with 3 isoforms, namely ERR $\alpha$ (NR3B1), ERR $\beta$ (NR3B2) and ERR $\gamma$ (NR3B3) (6-9). The 3 mammalian ERR genes have been implicated in diverse physiological processes, ranging from placental development to bone density 
maintenance $(10,11)$. The ERR $\alpha$ and ERR $\gamma$ isoforms have been demonstrated to perform core functions in the regulation of metabolic genes and cellular energy metabolism in skeletal muscle and heart (9-12). By contrast, the expression of ERR $\beta$ is only observed in restricted embryonic tissues (13), and its disruption causes fetal lethality resulting from impaired placentation (14). In addition, the post-natal expression of the ERR $\beta$ gene is highly restricted (15), therefore, less is known regarding its role in adult physiology (13). ERR $\alpha$ interacts with peroxisome proliferator-activated receptor $\gamma$ coactivator- $1 \alpha$ to stimulate vascular endothelial growth factor expression and angiogenesis in a hypoxia-inducible factor- $1 \alpha$-independent pathway following traumatic spinal cord injury (16). Genomic studies have demonstrated that ERR $\alpha$ and ERR $\gamma$ target a common set of gene promoters associated with fatty acid oxidation, oxidative phosphorylation and muscle contraction. Furthermore, due to the potential of overlapping target genes of ERR $\alpha$ and ERR $\gamma$ (17), the in vivo physiological importance of ERRs, particularly in neurons, remains to be determined.

Notably, in mice, experiments have revealed that ERR $\gamma$ deficiency accelerates the progression of pathologic processes and implicates the ERRs as etiological factors in diseases (18-20). In the central nervous system of mice, ERR $\gamma$ was highly expressed during neuronal differentiation (15). This transcription factor is also typically expressed at high levels in mature $\gamma$ but not $\alpha$ motor neurons of mice, forming a basis for distinguishing these 2 cell types $(5,20,21)$. Pei et al (20) also indicated that ERR $\gamma$ orchestrates the expression of a distinct neural gene network that promotes mitochondrial oxidative metabolism, thereby revealing the extraordinary neuronal dependence on glucose. In addition, ERR $\gamma$ defects in neuronal metabolism, particularly in mitochondrial oxidative phosphorylation, have been associated with ageing and diverse human neurological diseases (22). Results from gain- and loss-of-function models developed to characterize ERR function, and the use of small synthetic molecules to modulate their activity, have demonstrated the role of ERR in the control of skeletal muscle, heart and musculoskeletal physiology (9). Taken together, these data presented ERR $\gamma$ as a potential therapeutic target and a subject for further study, due to its co-localization with transcription factors involved in post-avulsion reactions. To the best of our knowledge, the pattern of expression of ERR $\gamma$ in the rat spinal cord, especially following BPRA, is unknown. Rats have often been selected as candidates for BPRA and spinal cord injury experiments, not only because they are readily available, but also due to their post-injury morphological, biochemical and functional changes that are similar to those observed in human patients (23). The present study aimed to explore the post-brachial plexus injury expression profile of the transcription factor ERR $\gamma$ and determine whether it may be used to define functionally distinct motor neuron sub-populations in the rat spinal cord.

\section{Materials and methods}

Animal model. A total of 35 adult female Sprague Dawley rats (weight, 180-250 g; age, 8-10 weeks) were purchased from the Laboratory Animal Centre of Sun Yat-sen University. The rats were housed under a 12-hour light/dark cycle, with ad libitum access to rat chow and water. All surgical procedures were conducted aseptically, in accordance with the Chinese National Health and Medical Research Council animal ethics guidelines. The experiments were approved by the Sun Yat-sen University Animal Experimentation Ethics Committee.

BPRA surgery. BPRA was performed as previously described $(24,25)$ In brief, the rats were anesthetized with a mixture of ketamine $(80 \mathrm{mg} / \mathrm{kg})$ and xylazine $(8 \mathrm{mg} / \mathrm{kg})$ administered intramuscularly (IM). While in the supine position, the right brachial plexus was exposed and identified, and its roots (C5-T1) were isolated under a dissecting microscope (magnification x10). Extra-vertebral avulsion of the ventral and dorsal roots was then performed. The ventral and dorsal roots, in addition to the dorsal root ganglia, were cut off at the distal ends of the avulsed spinal nerves and examined under the microscope to confirm the success of the surgery.

Retrograde labelling of the injured spinal motor neurons with fluorogold $(F G)$. A total of 3 days prior to BPRA surgery, FG retrograde labelling of the avulsion-injured motor neurons was performed on 5 adult SD rats; procedures were performed as previously described $(26,27)$. Briefly, the rats were anesthetized with a mixture of ketamine $(80 \mathrm{mg} / \mathrm{kg})$ and xylazine $(8 \mathrm{mg} / \mathrm{kg})$ (Fujian Gutian Pharmaceutical Co., Ltd.) administered IM, and laid in supine position under the dissecting microscope for surgery. Following the identification of the right brachial plexus, the $\mathrm{C} 7$ and $\mathrm{C} 8$ spinal nerve roots were injected with $2 \%$ FG ( $2 \% \mathrm{w} / \mathrm{v}$; Fluorochrome, LLC). A micropipette with the FG solution $(2.0 \mu \mathrm{l}$ for $60 \mathrm{sec})$ was slowly injected under the epineurium into the proximal stumps of the $\mathrm{C} 7$ and $\mathrm{C} 8$ nerve roots. The injection site was then clamped with micro forceps for an additional $10 \mathrm{sec}$ to ensure all of the axons had been cut. Finally, the muscle, fascia and skin were sutured successively in layers. Then, 3 days later, all rats in this group were anesthetized again. In prone position under the dissecting microscope, the rats underwent laminectomy of the C6 to C7 vertebrae, cutting of the dura mater and opening of the subarachnoid space to expose the dorsal and ventral roots of the $\mathrm{C} 7$ and $\mathrm{C} 8$ spinal nerves. Following positive identification, all of the dorsal and ventral rootlets of $\mathrm{C} 7$ and $\mathrm{C} 8$ were pulled out using a micropipette hook. Muscles, fascia and skin were then sutured successively in layers and the rats were allowed to survive for 3 days until sacrifice. Tissues were processed for FG and cyclic AMP-dependent transcription factor 3 (ATF-3) immunofluorescence as previously described (27).

Western blot preparation and analysis. To analyze the ERR $\gamma$ protein levels in the spinal cord segment that underwent root avulsion, 10 rats were sacrificed with a lethal dose of a mixture of ketamine $(320 \mathrm{mg} / \mathrm{kg})$ and xylazine $(32 \mathrm{mg} / \mathrm{kg})$ at $2(n=5)$ and 4 weeks $(n=5)$ following BPRA. Following confirmation of the absence of corneal and pain reflexes, the C7/C8 spinal segments were rapidly exposed, dissected and divided into left and right halves. The avulsed side was the right side, with the left side used as a control. Western blot analysis was performed as previously described (28). Samples (pooled left sides, right sides, and left and right of C7/C8 spinal segments of normal rats) were sonicated on ice in lysis buffer with $0.1 \%$ protease inhibitor and $0.5 \%$ PMSF to extract the total protein using the Total Protein Extraction Sample kit 
according to the manufacturer's protocol (Nanjing KeyGen Biotech Co., Ltd.). Protein concentration in each sample was determined using BCA protein assay kit, according to the manufacturer's protocol. The samples were diluted in an equal volume of 5X SDS loading buffer. The proteins in the samples $(40 \mu \mathrm{g})$ were then separated using a $10 \% \mathrm{TGX}^{\mathrm{TM}}$ FastCast $^{\mathrm{TM}}$ Acrylamide kit (Bio-Rad Laboratories, Inc.), transferred onto PVDF membranes and then blocked with $5 \%$ milk in TBST solution for $2 \mathrm{~h}$ at room temperature. Next, the PVDF membranes were probed with ERR3 (1:2,000; cat. no., sc66883, Santa Cruz Biotechnology, Inc) and GAPDH (1:2,000; cat. no., SAB1405848; Sigma-Aldrich: Merck KGaA) primary antibodies diluted in Western Blot Immune Booster solution 1 (Santa Cruz Biotechnology, Inc.) overnight at $4^{\circ} \mathrm{C}$. These membranes were then washed and probed with horseradish peroxidase-conjugated goat anti-mouse IgG (1:5,000; cat. no., AP127P; Merck KGaA) and anti-rabbit IgG (1:5,000; cat. no., AP107P; Merck KGaA) secondary antibodies for $2 \mathrm{~h}$ at room temperature, developed using enhanced chemiluminescence substrate and then exposed on BioMax film (Kodak). Exposed films were scanned, and protein bands quantified using Image-Pro Plus software version 6.0 (Media Cybernetics, Inc.).

Immunohistochemistry (IHC) and immunofluorescence (IF). The IHC and IF procedures were performed as previously described (27). Briefly, rats were deeply anesthetized with an intramuscular injection of a mixture of ketamine $(80 \mathrm{mg} / \mathrm{kg})$ and xylazine $(8 \mathrm{mg} / \mathrm{kg})$ at 2 (5 experimental rats and 5 control) and 4 weeks (5 experimental rats and 5 control) following BPRA surgery, and perfused transcardially with normal saline, followed by $4 \%$ paraformaldehyde in $0.1 \mathrm{M}$ PBS (pH 7.4). Following perfusion, the $\mathrm{C} 7$ and $\mathrm{C} 8$ spinal segments of each rat were carefully removed, immersed in fixative (4\% paraformaldehyde for $4 \mathrm{~h}$ at $4^{\circ} \mathrm{C}$ ) and then stored in $30 \%(\mathrm{v} / \mathrm{v})$ sucrose solution in PBS overnight. For IHC staining, frozen transverse sections $(35 \mu \mathrm{m})$ were washed 3 times with $0.01 \mathrm{M}$ PBS for $10 \mathrm{~min}$ and incubated in $0.3 \%$ peroxide in methanol $(100 \%)$ at room temperature for $15 \mathrm{~min}$ to eliminate endogenous peroxidase activity. Following washing in PBS, the sections were incubated in 3\% BSA (NeoFroxx $\mathrm{GmbH}$ ) and $0.3 \%$ Triton $\mathrm{X}-100$ in $0.01 \mathrm{M}$ PBS at room temperature for $30 \mathrm{~min}$ and then for $72 \mathrm{~h}$ at $4{ }^{\circ} \mathrm{C}$ with the following primary antibodies: Anti-ERR $\gamma$ (1:500; cat. no., sc66883; Santa Cruz Biotechnology, Inc.). Following washing in PBS, sections were incubated with the anti-rabbit IgG secondary antibody (1:5,000; cat. no., 31470; Invitrogen; Thermo Fisher Scientific, Inc.) at room temperature for $2 \mathrm{~h}$. The sections were then rinsed and incubated with ABC reagents (1:500; Wuhan Boster Biological Technology, Ltd.) at room temperature for $45 \mathrm{~min}$. The sections were then washed thoroughly and incubated in 0.05 DAB (Nanjing KeyGen Biotech Co., Ltd.) and $0.01 \%$ $\mathrm{H}_{2} \mathrm{O}_{2}$ for 3-5 min until a brown reaction product was observed.

For IF double-labelling, sections from 5 rats were incubated in 3\% BSA and $0.3 \%$ Triton X-100 in 0.01 M PBS at room temperature for $30 \mathrm{~min}$ and incubated with the following primary antibodies for $72 \mathrm{~h}$ at $4^{\circ} \mathrm{C}$ : Anti- Neuronal Nuclei (1:500; cat. no., ab104224; Abcam); anti-ERR $\gamma$ (1:500; cat. no., sc66883; Santa Cruz Biotechnology, Inc.); and anti-ATF-3 (1:500; cat. no., sc81189; Santa Cruz Biotech Inc.). Following washing in PBS, the sections were incubated with fluorescein isothiocyanate-conjugated anti-mouse $\operatorname{IgG}(1: 1,000$; cat. no., F-2761; Invitrogen; Thermo Fisher Scientific, Inc.), TRITC-conjugated anti-Rabbit IgG (1:1,000; cat. no., A18750; Invitrogen; Thermo Fisher Scientific, Inc.) at room temperature for $2 \mathrm{~h}$ in the dark. The sections were washed again in PBS, mounted on glass slides and examined under a fluorescence microscope at x10 and x20 magnification (Zeiss AG). The staining specificity was verified by the omission of primary antibodies.

The mean of a total number of ERR $\gamma$-positive motor neurons in 10 serial IHC sections of the ventral horns of C7 and $\mathrm{C} 8$ spinal segment was calculated in each rat. The positive motor neurons, the ones exhibiting visibly stained nuclei of the spinal cord, were counted under a microscope at a magnification, x20 (Carl Zeiss AG) as described previously $(29,30)$. Enumeration of motor neurons, pooling of means and data analysis were performed by two independent persons blinded to the treatment/sidedness of the groups.

Statistical analysis. Enumeration of motor neurons, pooling of means and data analysis were performed by two independent persons blinded to the treatment sidedness of the groups. The data are presented as the mean \pm standard error of the mean and were analyzed using SPSS v.16.0 software (SPSS, Inc.). A one-way analysis of variance was used to analyze the differences among groups, followed by a post-hoc Bonferroni test. $\mathrm{P}<0.05$ was considered to indicate a statistically significant difference.

\section{Results}

Avulsion-induced ATF-3 is a marker of injured motor neurons. At the end of the 6 days after the FG retrograde labelling, the C7 and C8 spinal cord sections from 5 rats were examined under a fluorescence microscope. The results indicated that motor neurons in the ipsilateral ventral horn were labelled by FG (Fig. 1). In addition, almost all motor neurons, including their cell bodies and their dendritic processes, were labelled by the FG in the ipsilateral $\mathrm{C} 7$ and $\mathrm{C} 8$ ventral horns (Fig. 1Aa). In the FG-labelled motor neurons, the FG dye was taken up by the cut end of the axons in the middle and lower trunks of the brachial plexus and then transported to the cytoplasm of the motor neuron cell bodies in the $\mathrm{C} 7$ and 8 ventral horn segments via retrograde transport (Fig. 1). Therefore, FG-labelled motor neurons represented the injured motor neurons, whose axons had been severed. Fig. $1 \mathrm{Bb}$ also indicated that, on day 3 following avulsion, ATF-3 was expressed in the nuclei of motor neurons whose axons had been severed by root avulsion. It was, therefore, only expressed in the ipsilateral ventral horn of the spinal cord corresponding to the level of the root avulsion. Fig. 1Cc additionally demonstrates the co-localization of FG with ATF-3, thereby providing evidence that the ATF-3 is a viable marker of injured motor neurons.

Avulsion decreases ERR $\gamma$ protein expression in the ipsilateral half of injured spinal cords. The expression of the ERR $\gamma$ protein in the spinal cord following root avulsion was assessed by western blot analysis using an anti-ERR $\gamma$ antibody. At each time point [ 2 weeks $(n=5)$ and 4 weeks $(n=5)]$, the results of the 
A

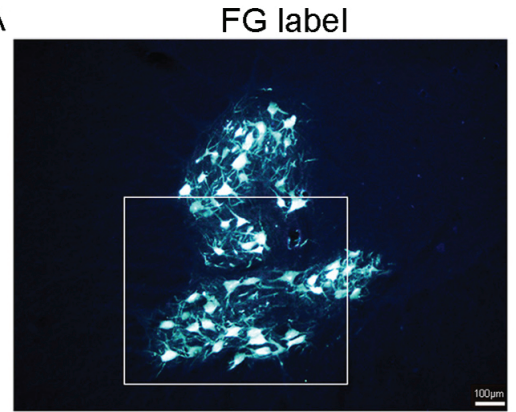

a
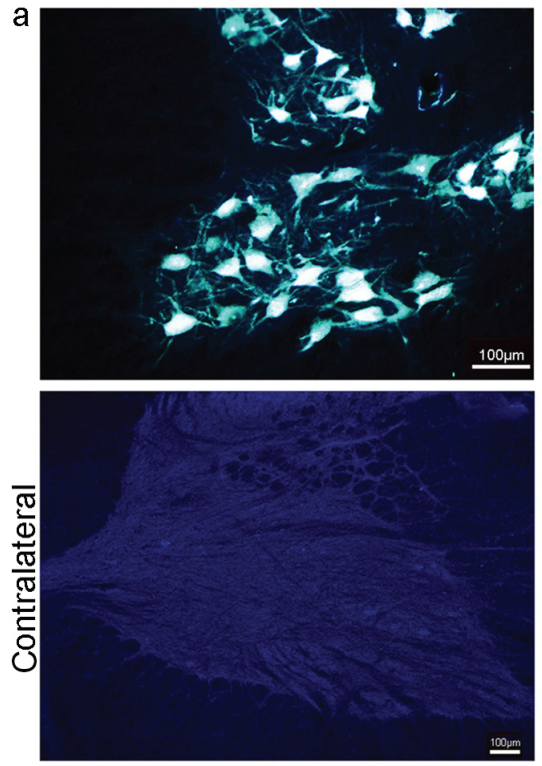

D
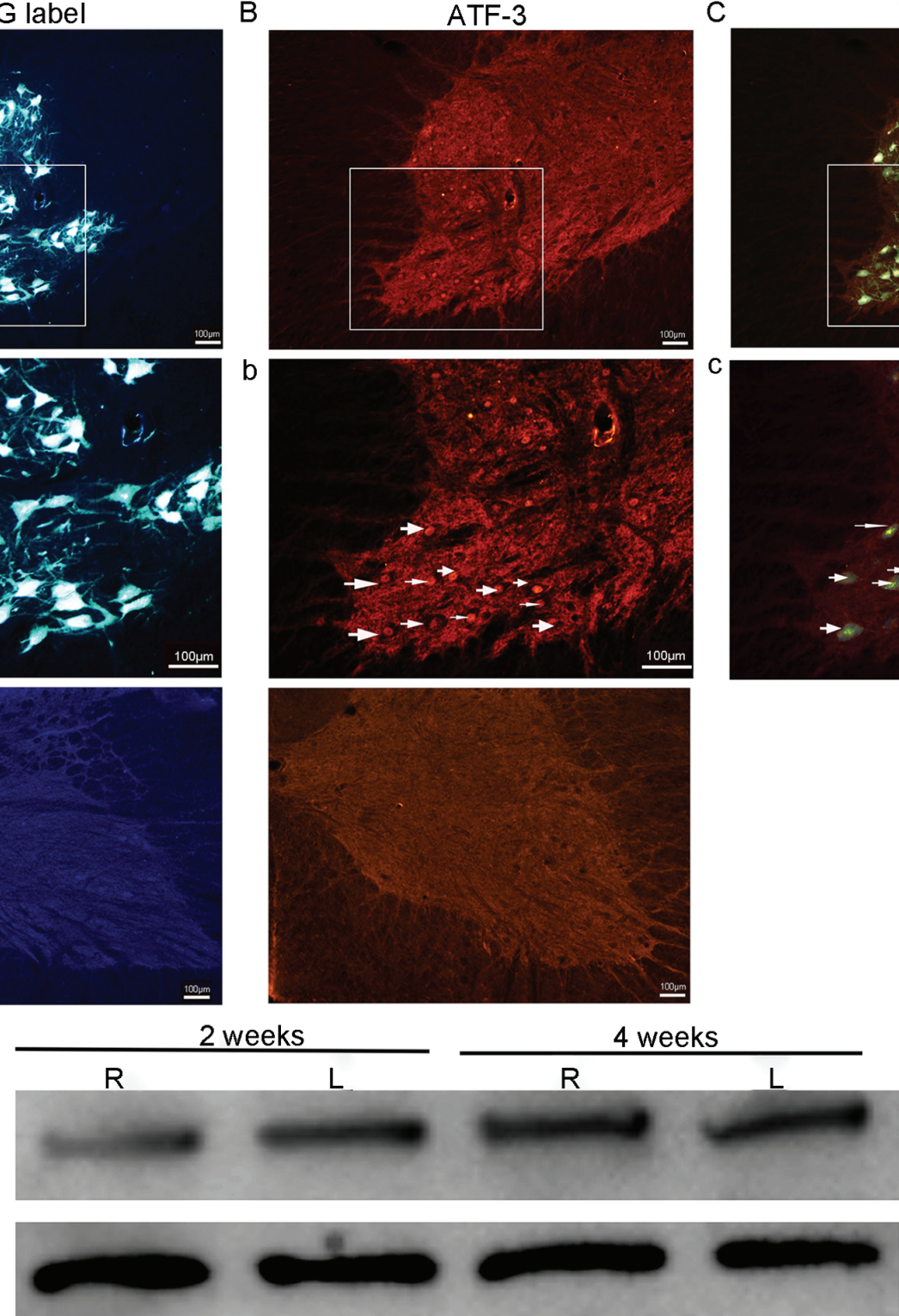

$\mathrm{b}$
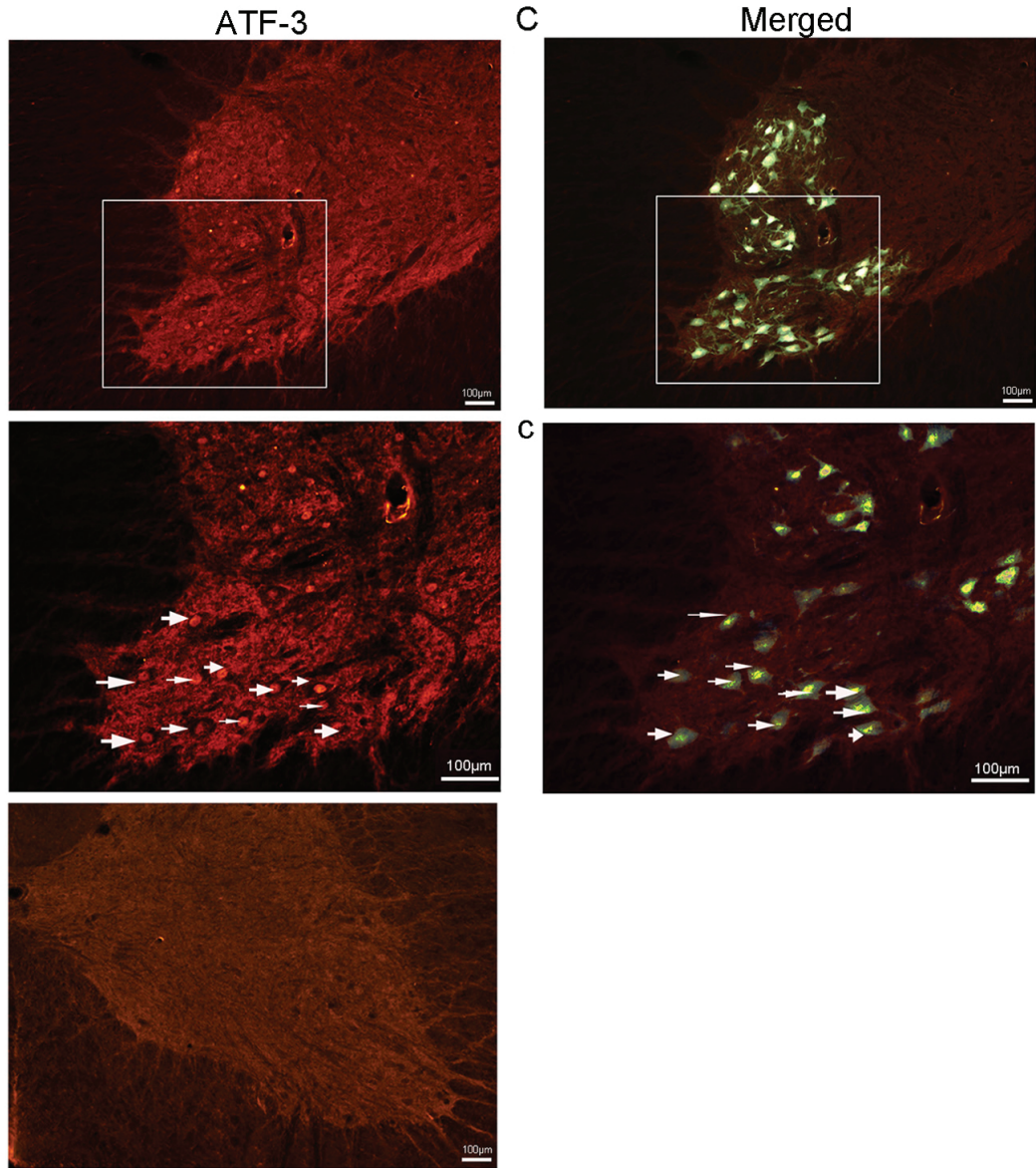

4 weeks

R $\quad$ L

$51 / 49 \mathrm{kDa}$

$36 \mathrm{kDa}$

E

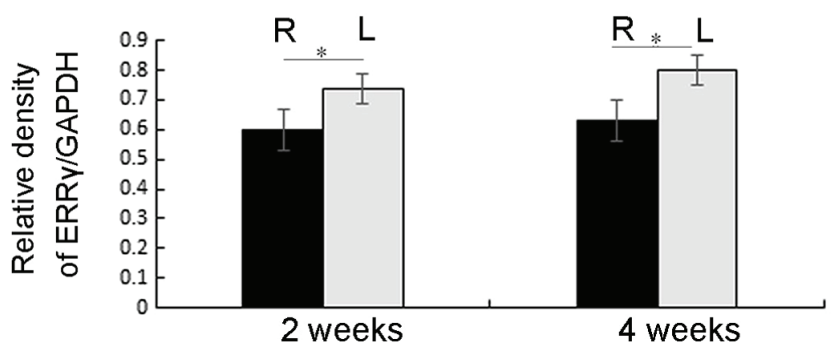

Figure 1. FG labeling and ATF-3/ERR $\gamma$ in the injured spinal cord. FG retrograde labeling was performed in the representative axotomy side C7 and C8 spinal cord sections examined under the fluorescence microscope at day 3. (A) FG localization in the cell somata and cell processes of neurons whose axons were cut at the level of the brachial plexus roots. Scale bar=100 $\mu \mathrm{m}$, magnification x10. (a) Magnification (x20) of the area marked in image A. Scale bar=100 $\mu \mathrm{m}$. (B) AFT-3 nuclei staining on the axotomized side of the C7 and C8 spinal cord sections examined under the fluorescence microscope at day 3. No staining was observed on the contralateral side (not in view). Magnification x10 (b). Magnification (x20) of the area marked in image B. Scale bar=100 $\mu \mathrm{m}$. (C) A merged image demonstrating co-localization of FG and ATF-3 in injured (axotomy) motor neurons at day 3. Magnification x10. (c) Magnification (x20) of the area marked in image C. Scale bar=100 $\mu \mathrm{m}$. (D) A representative western blot analysis gel. The ERR $\gamma /$ GAPDH ratio for the L (uninjured side) and R (injured side) halves of the spinal cord of each at 2 and 4 weeks post-avulsion. (E) Densitometry results are presented as means \pm standard error of the mean. The level of ERR $\gamma$ protein was significantly decreased on the right side (brachial plexus injured) of the spinal cord at both time points. "P<0.05. The black bars represent the protein level on the injured side, and the gray bars represent the expression levels on the contralateral, uninjured side. FG, Fluorogold; ATF-3, cyclic AMP-dependent transcription factor 3; ERR $\gamma$, estrogen-related receptor $\gamma$; L, left, contralateral side; R, right ipsilateral side.

representative western blot analysis suggested that the ERR $\gamma$ protein level in the ipsilateral half, the avulsed half of the spinal cord, was significantly decreased compared with that of the contralateral half (all P<0.05; Fig. 1D and E). 
A

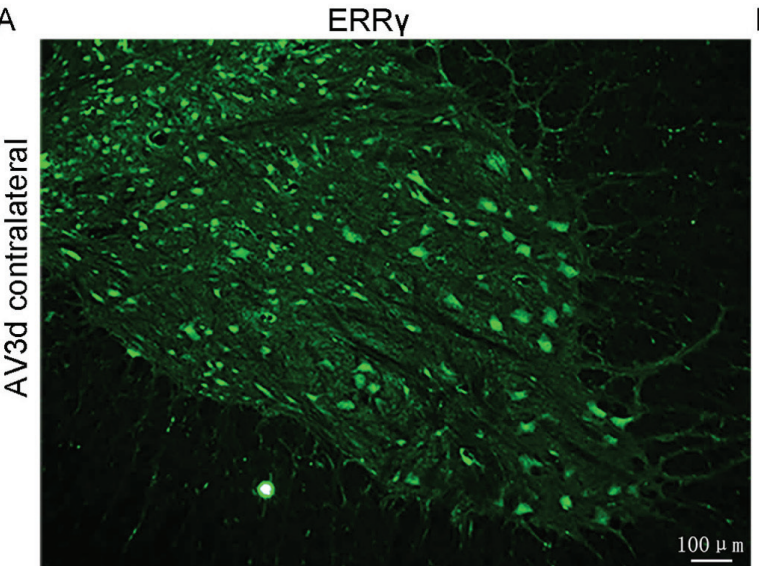

B ATF-3

C
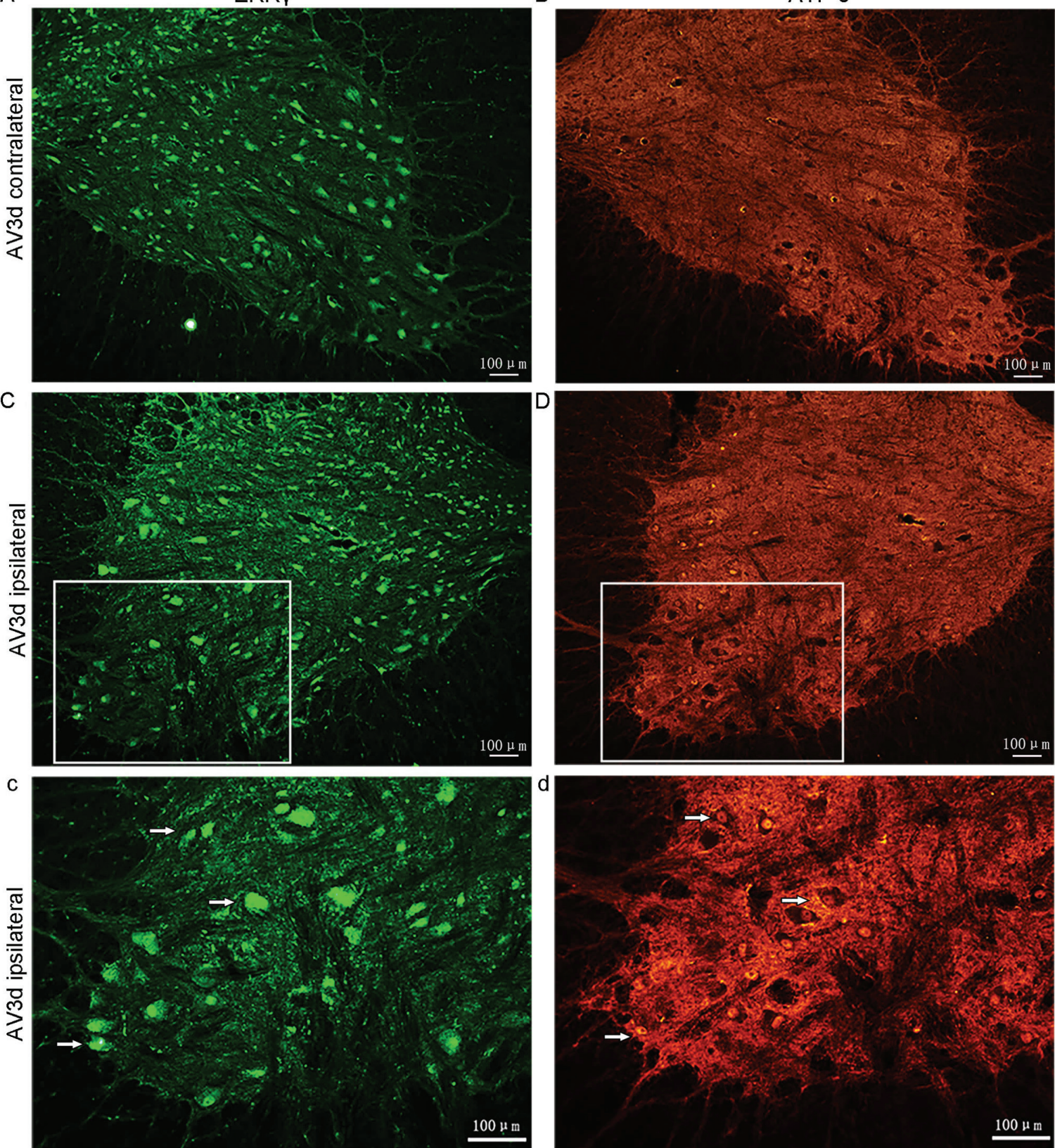

Figure 2. Expression of ERR $\gamma$ and ATF-3 in ventral horn motor neurons. Adjacent sections were either stained with ERR $\gamma$ or ATF-3 antibodies. (A) ERR $\gamma$ expression on the contralateral ventral horns 3 days following avulsion. (B) No ATF-3 positive neurons on the contralateral ventral horns of the C7 and C8 were observed 3 days following avulsion. (C) Downregulation of ERR $\gamma$ expression on the ipsilateral side. (c) Magnification, $x 20$ of the area marked in image C. Scale bar=100 $\mu \mathrm{m}$. (D) ATF-3 was detected on the ipsilateral side of corresponding segments in the same population of motor neurons as in the ERR $\gamma$-stained sections. (d) Magnification, x 20 of the area marked in image D. Scale bar=100 $\mu \mathrm{m}$. ATF-3, cyclic AMP-dependent transcription factor 3; ERR $\gamma$, estrogen-related receptor $\gamma$.

Avulsion-induced death of the ERR $\gamma$ and ATF-3 positive motor neurons. In the ERR $\gamma$-stained sections, the numbers of positive motor neurons were notably decreased in the ipsilateral side compared with those in the contralateral side (Fig. 2A-Cc). The data also demonstrated that the positive signal for ERR $\gamma$ was present in both small and large motor neurons. These results, therefore, demonstrated that there was constitutive expression of ERR $\gamma$ and that BPRA induced $E R R \gamma$ downregulation in motor neurons in the injured segment of the spinal cord.

In addition, the expression of ATF-3 in the spinal cord following root avulsion was also assessed using IF. Adjacent sections were either stained with an antibody against ERR $\gamma$ or ATF-3. At 3 days following avulsion, no ATF-3 positive neurons were identified on the contralateral ventral horns of the $\mathrm{C} 7$ and C8 $(\mathrm{n}=5)$. However, ATF-3 was detected on the ipsilateral side of corresponding segments (Fig. 2B-Dd) $(n=5)$.

In Fig. 3 , the representative IHC images 2 weeks post-avulsion indicate fewer ERR $\gamma$-positive motor neurons in the ipsilateral injured side (Fig. 3B) compared with in the contralateral side (Fig. 3A). This trend was also observed 4 weeks post-avulsion (Fig. 3C and D). The ERR $\gamma$-positive signal was present in both smaller $\gamma$ (red arrow) and larger $\alpha$ (green arrow) motor neurons. The results of immunoreaction cell counting in 

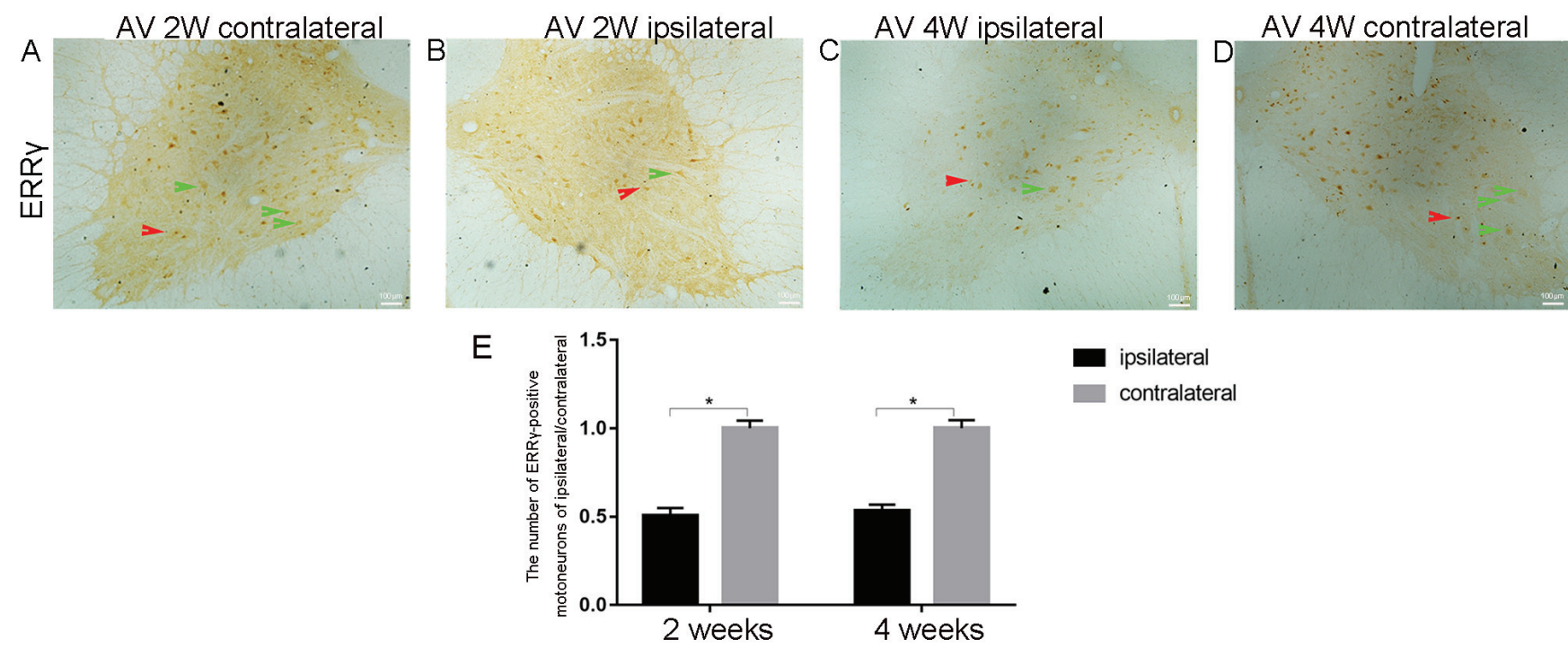

Figure 3. Representative micrographs used for the counting of the ERR $\gamma$ positive motor neurons on both sides of the same C7 segment of the spinal cord. (A) The contralateral side at 2 weeks. (B) The ipsilateral side at 2 weeks. (C) The ipsilateral side at 4 weeks. (D) The contralateral side at 4 weeks; all at magnification $\mathrm{x} 20$ ) In the ERR $\gamma$-stained sections, the numbers of positive motor neurons were notably decreased on the ipsilateral side compared with those on the contralateral side. Red arrows show positive ERR $\gamma$ signals in smaller $\gamma$ motor neurons; Green arrows depict positive ERR $\gamma$ signals in large $\alpha$ motor neurons. (E) The mean of the total number of ERR $\gamma$-positive motor neurons in 10 serial sections of both ventral horns of C7 spinal segments were computed at 2 and 4 weeks post-avulsion. The average number of ERR $\gamma$-positive motor cells on the injured side (black column, ipsilateral) were significantly decreased compared with that identified on the contralateral side (gray column, contralateral). ${ }^{*} \mathrm{P}<0.05$. ERR $\gamma$, estrogen-related receptor $\gamma$.

the ventral horns demonstrated that the average number of the ERR $\gamma$-positive neurons in the ipsilateral ventral horn on day 14 was $9 \pm 1(n=5)$ and on day 28 was $11 \pm 0.5(n=5)$ (Fig. 3E). On the contralateral ventral horn, the number of ERR $\gamma$-positive neurons on day 14 was $18 \pm 1$, while on day 28 it was $21 \pm 1.5$ (Fig. 3E). Further observations and statistical analysis indicated that avulsion significantly decreased the number of ERR $\gamma$-positive motor neurons in the ipsilateral ventral horn, both on days 14 and 28 compared with the number in the contralateral ventral horn (all $\mathrm{P}<0.05$; Fig. 3E).

ERR $\gamma$ nonspecific expression in $\gamma$ and $\alpha$ motor neurons. In mice, $\gamma$ and $\alpha$ motor neurons were distinguished based on the expression of ERR $\gamma(5)$. In the present study involving a rat model, the IF double-labelling reaction results indicated that the positive immunoreactions of the NeuN antibody ERR $\gamma$ (red, Fig. 4A-D) and (green, Fig. 4E-H) were concentrated in the cytoplasm and nuclei of the spinal neurons in the contralateral ventral horns of the injured spinal segments, respectively. The area that was used for cell counting is marked in Fig. 4G. Almost all ERR $\gamma$-positive motor neurons were also NeuN-positive (Fig. 4I-L). However, a few motor neurons were $\mathrm{ERR} \gamma^{\text {on }} / \mathrm{NeuN}^{\text {off }}$. These results demonstrated that the ERR $\gamma^{\text {on }} / \mathrm{NeuN}^{\text {off }}$ status of motor neurons can be identified as $\gamma$ motor neurons (Fig. 4M). In addition, the presence of $\mathrm{ERR} \gamma^{\text {on }} / \mathrm{Neu} \mathrm{N}^{\text {off }}$ implies that ERR $\gamma$ was not specific to a single cell subtype, the $\gamma$ motor neuron.

\section{Discussion}

The aim of the present study was to describe the post-brachial plexus avulsion expression profile of ERR $\gamma$ and determine whether ERR $\gamma$ and NeuN have complementary expression profiles in $\gamma$ and $\alpha$ motor neurons.
In a previous study, ATF-3-positive immunoreaction was demonstrated to be a marker of neuronal survival and regenerative competency following experimental avulsion injury and, as such, the authors suggested the use of ATF-3 as a regenerative marker of the affected motor neurons (31). In the present study, ATF-3 was not expressed on the intact half of the spinal cord (the contralateral side), but was markedly induced in the avulsion side of the spinal motor neurons of the corresponding cord segments. The FG-labelled motor neurons represented the injured motor neurons, as these FG-labelled motor neuron somata received the FG tracer that had been taken up and retrogradely transported by the remaining stumps of the axons. These results were in concordance with our previous study (27). ATF-3 expression also occurred only in the nuclei of the FG-positive neurons, as previously demonstrated (27). In previous studies, ATF-3 was revealed to be induced in the liver upon intoxication or hepatectomy, in the heart upon myocardial ischemia or ischemia-reperfusion, and in the kidney upon renal ischemia-reperfusion $(32,33)$. In each of these cases, including those in our studies, ATF-3 was notably and consistently induced in the corresponding tissues that are exposed to the stress signal. Therefore, ATF-3 may serve an important role in the general early response to stress. The present data suggested that ATF-3 may be a specific phenotypic marker of BPRA-injured motor neurons, as BPRA resulted in an early and sustained expression of ATF-3 in the injured spinal motor neurons. In the ipsilateral spinal cord horn, avulsion induced a sustained expression of ATF-3 in the nuclei.

In the present study, it was identified that there was a consistent decrease in the levels of ERR $\gamma$ protein expression on the brachial plexus cord avulsion-injured side of the spinal cord. These results were consistent with the other results of the present study, indicating that the number of ERR $\gamma$-positive motor neurons was also decreased in the same injured side 

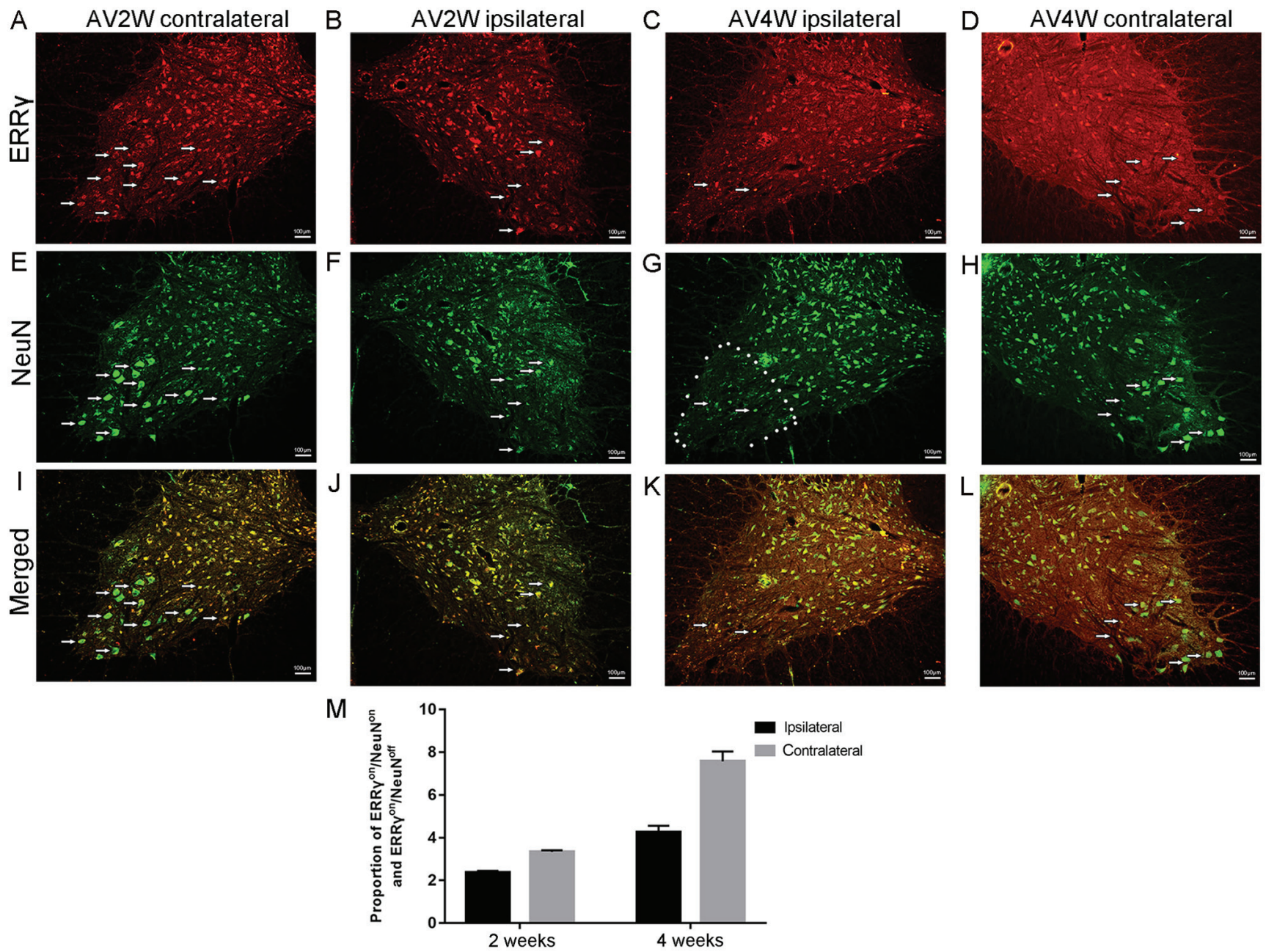

Figure 4. Complementary expression of ERR $\gamma$ and NeuN in $\gamma$ and $\alpha$ motor neurons. Immunofluorescence double-labeling reaction results indicated that the positive immunoreactions at (A) 2 weeks in the contralateral side of the spine, (B) 2 weeks in the ipsilateral side of the spine, (C) 4 weeks in the ipsilateral side of the spine and (D) 4 weeks in the contralateral side of the spine. ERR $\gamma$ (red) were located in the cytoplasm of the spinal neurons. The figures also show the apparent downregulation of the ERR $\gamma$ signal on the injured ipsilateral sides at the time points after avulsion. NeuN (green) signals were concentrated in the nuclei of motor neurons as shown after (E) 2 weeks in the contralateral side of the spine, (F) 2 weeks in the ipsilateral side of the spine, (G) 4 weeks in the ipsilateral side of the spine and (H) 4 weeks in the contralateral side of the spine. Representative micrographs after (I) 2 weeks in the contralateral side of the spine, (J) 2 weeks in the ipsilateral side of the spine, (K) 4 weeks in the ipsilateral side of the spine and (L) 4 weeks in the contralateral side of the spine show that the bulk all of the ERR $\gamma$-positive motor neurons were also NeuN-positive motor cells (ERR $\gamma^{\text {on }} / \mathrm{NeuN}^{\mathrm{on}}$ ). However, the remainder of these ventral motor neurons were ERR $\gamma^{\text {on }} / \mathrm{NeuN}^{\text {off }}$. (M) ERR $\gamma$ was detected in both populations of motor neurons $\mathrm{ERR} \gamma^{\text {on }} / \mathrm{NeuN}^{\text {off }}$ status of motor neurons can be identified as $\gamma$ motor neurons. ERR $\gamma$, estrogen-related receptor $\gamma$; NeuN, RNA binding protein fox-1 homolog 3.

over the same observation period. In combination, these results indicated that the effects of brachial plexus injury may have led to the death of ERR $\gamma$-positive motor neurons. This result was not in agreement with earlier data that $\gamma$ motor neurons are largely spared following secondary spinal cord injury $(34,35)$. In a previous study $(34)$, the authors postulated that the surviving $\gamma$ motor neurons may have indirectly exacerbated the death of motor neurons through a regimen of excitotoxic proprioceptive afferent (IA) feedback on $\alpha$ motor neurons. Although the model in the present study was not able to molecularly distinguish $\gamma$ from $\alpha$ motor neurons, and there may also be confounding caused by a decrease in the size of the dying $\alpha$ motor neurons; future studies should focus on the identification of these subtypes to determine whether this phenomenon may be involved in present neuronal cell loss. In addition, well-established causes of motor neuron loss following avulsion injuries, such as nitric oxide $(25,28,36)$ or JNK-mediated (phosphorylated c-Jun) apoptosis (37) could not be ruled out. It could be suggested that, in this case, all axons of the motor neuron pool under study were severed, therefore, the interaction whereby $\gamma$ causes $\alpha$ motor neuron degeneration could only happen at the level of the spinal cord ventral horn. This is an additional avenue for further study.

ERRs, the first orphan nuclear receptors, share sequence homology with members of the nuclear receptor superfamily $(38,39)$. Studies have identified that the ERRs control vast metabolic gene networks and are key regulators of energy metabolism, particularly in response to various environmental challenges or biological stresses (13). However, results from multiple genome-wide binding site location analyses have suggested that ERRs may also be major orchestrators of other biosynthetic pathways and biological processes; furthermore, this regulation is likely to be cell- and tissue-specific. ERR $\gamma$ was the first orphan receptor to be identified due to its interaction with transcriptional coactivators. In a number of respects, ERR $\gamma$ functions like a classical nuclear receptor. Although it 
has been demonstrated that ERR $\gamma$ is a functional transcriptional activator, its true physiological role remains to be determined. The highest expression of ERR $\gamma$ occurs around days 11-15 of mouse embryonic development, a period of very active organogenesis (15). ERR $\gamma$ was also expressed in selected adult tissues such as the heart, kidney and muscle (40). ERR $\gamma$ has roughly similar temporal patterns of expression in mouse embryos, and somewhat similar- although not identical- distributions in adult tissues $(15,27,41)$. The transcription factor ERR $\gamma$ is expressed at high levels in $\gamma$ but not $\alpha$ motor neurons, whereas the neuronal DNA binding protein, NeuN, marks $\alpha$ but not $\gamma$ motor neurons in mice (5). The present study aimed to determine whether $\gamma$ and $\alpha$ motor neurons in the spinal cord of the rats are distinguishable on the basis of their profile of expression of transcription factors and other molecular markers, as previously reported in mice (5). In this previous study, both the distribution and frequency of small, ERR positive/RNA binding protein fox-1 homolog $3(\mathrm{NeuN})$ negative (ERR $\gamma$ on/NeuNoff) motor neurons in this previous study matched the profile expected for $\gamma$ motor neurons (5). Results from the present study demonstrated that ERR $\gamma_{\text {on }} / \mathrm{NeuN}_{\text {off }}$ neurons could be assumed to be $\gamma$ motor neurons; however, not all ERR $\gamma_{\text {on }}$ (positive) neurons were $\gamma$ motor neurons. Even the largest neurons in the ventral horn ( $\alpha$ motor neurons) also exhibited the ERR $\gamma$ signal in the present study. In light of the above results, further studies are required to establish those transcription factors that may be used to mark and positively distinguish between motor neuron subtypes within the motor neuron pool. In the present study, it was apparent that ERR $\gamma$ was promiscuous, and therefore did not qualify as a molecular marker, as it did in mice (5).

The data from the present study proposed the relevance of ERR $\gamma$ in mediating motor neuron response to avulsion-associated stress. It was apparent that the ERR $\gamma$ expression level was decreased on the injured side of the spinal cord, indicating that it may participate in certain response-to-injury signaling pathways or have a constitutive expression role in normal spinal cord cells. To the best of our knowledge, the present results provided the first insights into the role of ERR $\gamma$ in the spinal cord, as a novel approach toward understanding specific motor neuron response to BPRA stress. We hypothesized that a decrease in the ERR $\gamma$ expression, specifically in the injured motor neurons that eventually died, may imply a neuroprotective role should it have been present. However, further studies are required to verify the exact role of ERR $\gamma$. It is well established that the neuroendocrine pathways that regulate gonadotropin release in rodents are sexually dimorphic and profoundly affected by neonatal estrogens (42). It would be important and interesting for future studies to explore the differences in ERR expression following BPRAs and elucidate their co-localization a well as roles in neuroprotection and neurodegeneration, if any.

In conclusion, the data of the present study on ERR $\gamma$ expression demonstrated that $\gamma$ and $\alpha$ motor neurons cannot be distinguished molecularly, and, as a result, there are no complementary profiles of DNA binding protein and ERR $\gamma$ expression. Therefore, contrary to previous data from mice (5), the principle that spinal motor neurons may be fractionated into functionally distinct subtypes on the basis of their profile of transcription factors (ERR $\gamma$ and NeuN) does not appear to extend to neuronal subtypes within single motor neuron pools in Sprague Dawley rats. Instead, in rats, there is at least a tendency that ERR $\gamma$ and $\mathrm{NeuN}$ are expressed in the same subpopulations of motor neurons in the rat spinal cord, due to the observed expression promiscuity. However, the downregulation of ERR $\gamma$ in the injured side of the spinal cord is an intriguing result that should be investigated further.

\section{Acknowledgements}

The authors would like to thank Professor Fu Rao of the Anatomy Department of Sun Yat-sen University, China for general scientific review of the manuscript.

\section{Funding}

The present study was supported by a research grant from the National Science Foundation Council of China (grant no. 31471030$)$.

\section{Availability of data and materials}

All data generated or analyzed during this study are included in this published article.

\section{Authors' contributions}

LHZ conceptualised and designed the study. GY, PLMZ and LL performed the experiments. KZ, YT and ZL assisted with some of the experiments, and performed data analysis and cell counting. GY and PLMZ wrote the manuscript draft, all authors revised it and then PLMZ made final edits to the paper. All authors read and approved the final manuscript.

\section{Ethics approval and consent to participate}

Ethical approval was obtained from the Sun Yat-sen University Animal Experimentation Ethics Committee prior to the execution of the present study. This study was conducted in accordance with the Chinese National Health and Medical Research Council (NHMRC) animal ethics guidelines.

\section{Patient consent for publication}

Not applicable.

\section{Competing interests}

The authors declare that they have no competing interests.

\section{References}

1. Lu Y, Liu H, Hua X, Xu WD, Xu JG and Gu YD: Supplementary motor cortical changes explored by resting-state functional connectivity in brachial plexus injury. World Neurosurg 88: 300-305, 2016.

2. Noristani HN, Sabourin JC, Boukhaddaoui H, Chan-Seng E, Gerber YN and Perrin FE: Spinal cord injury induces astroglial conversion towards neuronal lineage. Mol Neurodeger 11: 68-81, 2016.

3. Stifani N: Motor neurons and the generation of spinal motor neuron diversity. Front Cell Neurosci 8: 293-302, 2014.

4. Manuel M and Zytnicki D: Alpha, beta and gamma motoneurons: Functional diversity in the motor system's final pathway. J Integr Neurosci 10: 243-276, 2011 
5. Friese A, Kaltschmidt JA, Ladle DR, Sigrist M, Jessell TM and Arber S: Gamma and alpha motor neurons distinguished by expression of transcription factor ERR3. Proc Natl Acad Sci USA 106: 13588-13593, 2009.

6. Tremblay AM and Giguère V: The NR3B subgroup: An overview. Nucl Recept Signal 5: e009, 2009.

7. Giguère V: Transcriptional control of energy homeostasis by the estrogen related receptors. Endocr Rev 29: 677-696, 2008.

8. Deblois $\mathrm{G}$ and Giguère V: Functional and physiological genomics of estrogenrelated receptors (ERRs) in health and disease. Biochim Biophys Acta 1812: 1032-1040, 2011.

9. Huss JM, Garbacz WG and Xie W: Constitutive activities of estrogen-related receptors: Transcriptional regulation of metabolism by the ERR pathways in health and disease. Biochim Biophys Acta 1852: 1912-1927, 2015.

10. He H, Xi G and Lu X: Molecular cloning, characterization, and expression analysis of an estrogen receptor-related receptor homologue in the cricket, Teleogryllus emma. J Insect Sci 10: 188-196, 2010

11. Yoshihara E, Wei Z, Lin CS, Fang S, Ahmadian M, Kida Y Tseng T, Dai Y, Yu RT, Liddle C, et al: ERR $\gamma$ is required for the metabolic maturation of therapeutically functional glucose-responsive $\beta$ cells. Cell Metab 23: 622-634, 2016.

12. Deblois G and Giguère V: Oestrogen-related receptors in breast cancer: Control of cellular metabolism and beyond. Nat Rev Cancer 13: 27-36, 2013.

13. Audet-Walsh É, and Giguère V: The multiple universes of estrogen-related receptor alpha and gamma in metabolic control and related diseases. Acta Pharmacol Sin 36: 51-61, 2015.

14. Luo J, Sladek R, Bader JA, Matthyssen A, Rossant J and Giguère V: Placental abnormalities in mouse embryos lacking the orphan nuclear receptor ERR- $\beta$. Nature 388: 778-782, 1997.

15. Bonnelye E, Vanacker JM, Spruyt N, Alric S, Fournier B, Desbiens X and Laudet V: Expression of the estrogen-related receptor 1 (ERR-1) orphan receptor during mouse development. Mech Dev 65: 71-85, 1997.

16. Hu JZ, Long H, Wu TD, Zhou Y and Lu HB: The effect of estrogen-related receptor $\alpha$ on the regulation of angiogenesis after spinal cord injury. Neuroscience 290, 570-580, 2015

17. Dufour CR, Wilson BJ, Huss JM, Kelly DP, Alaynick WA, Downes M, Evans RM, Blanchette $M$ and Giguere V: Genome-wide orchestration of cardiac functions by the orphan nuclear receptors ERR $\alpha$ and $\gamma$. Cell Metab 5: 345-356, 2007.

18. Kwon DH, Eom GH, Kee HJ, Nam YS, Cho YK, Kim DK Koo JY, Kim HS, Nam KI, Kim KK, et al: Estrogen-related receptor gamma induces cardiac hypertrophy by activating GATA4. J Mol Cell Cardiol 65: 88-97, 2013.

19. Murray J, Auwerx J and Huss JM: Impaired myogenesis in estrogen-related receptor $\gamma($ ERR $\gamma)$-deficient skeletal myocytes due to oxidative stress. FASEB J 27: 135-150, 2013.

20. Pei L, Mu Y, Leblanc M, Alaynick W, Barish GD, Pankratz M, Tseng TW, Kaufman S, Liddle C, Yu RT, et al: Dependence of hippocampal function on ERR $\gamma$-regulated mitochondrial metabolism. Cell Metab 21: 628-636, 2015.

21. Kida YS, Kawamura T, Wei Z, Sogo T, Jacinto S, Shigeno A, Kushige H, Yoshihara E, Liddle C, Ecker JR, et al: ERRs mediate a metabolic switch required for somatic cell reprogramming to pluripotency. Cell Stem Cell 16: 547-555, 2015.

22. Mattson MP, Gleichmann M and Cheng A: Mitochondria in neuroplasticity and neurological disorders. Neuron 60: 748-766, 2008.

23. Fleming JC, Norenberg MD, Ramsay DA, Dekaban GA, Marcillo AE, Saenz AD, Pasquale-Styles M, Dietrich WD, Weaver LC: The cellular inflammatory response in human spinal cords after injury. Brain 129: 3249-3269, 2006

24. Zhou LH, Han S, Xie YY, Wang LL and Yao ZB: Differences in c-jun and nNOS expression levels in motoneurons following different kinds of axonal injury in adult rats. Brain Cell Biol 36 213-227, 2008

25. Wu W,LiuzziFJ, Schinco FP, Depto AS, Li Y,Mong JA, Dawson TM and Snyder SH: Neuronal nitric oxide synthase is induced in spinal neurons by traumatic injury. Neurosci 61: 719-726, 1994.
26. Fu R, Tang Y, Ling ZM, Li YQ, Cheng X, Song FH, Zhou LH and $\mathrm{Wu} \mathrm{W}$ : Lithium enhances survival and regrowth of spinal motoneurons after ventral root avulsion. BMC Neurosci 15: 84-91, 2014

27. Tang Y, Ling ZM, Fu R, Li YQ, Cheng X, Song FH, Luo HX and Zhou LH: Time-specific microRNA changes during spinal motoneuron degeneration in adult rats following unilateral BPRA: Ipsilateral vs. contralateral changes. BMC Neurosci 15: 92-102, 2014

28. Wang J, Yan L, Zhao X, Wu W and Zhou LH: The diversity of nNOS gene expression in avulsion-injured spinal motoneurons among laboratory rodents. Nitric Oxide 22: 37-42, 2010.

29. Zhou L and Wu W: Antisense oligos to neuronal nitric oxide synthase aggravate motoneuron death induced by spinal root avulsion in the adult rat. Exp Neurol 197: 84-92, 2006.

30. Li YQ, Tang Y, Fu R, Meng QH, Zhou X, Ling ZM, Cheng X, Tian SW, Wang GJ, Liu XG and Zhou LH: Efficient labeling in vitro with non-ionic gadolinium magnetic resonance imaging contrast agent and fluorescent transfection agent in bone marrow stromal cells of neonatal rats. Mol Med Rep 12: 913-920, 2015.

31. Linda H, Skold MK and Ochsmann T: Activating transcription factor 3, a useful marker for a regenerative response after nerve root injury. Front Neurol 2: 30-38, 2011.

32. Chen BP, Wolfgang CD and Hai T: Analysis of ATF3, a transcription factor induced by physiological stresses and modulated by gadd153/Chop10. Mol Cell Biol 16: 1157-1168, 1996.

33. Yin T, Sandhu G, Wolfgang CD, Burrier A, Webb RL, Rigel DF, Hai T and Whelan J: Tissue-specific pattern of stress kinase activation in ischemic/reperfused heart and kidney. J Biol Chem 272: 19943-19950, 1997.

34. Powis RA and Gillingwater TH: Selective loss of alpha motor neurons with sparing of gamma motor neurons and spinal cord cholinergic neurons in a mouse model of spinal muscular atrophy. J Anat 228: 443-451, 2016.

35. Lalancette-Hebert M, Sharma A, Lyashchenko AK and Shneider NA: Gamma motor neurons survive and exacerbate alpha motor neuron degeneration in ALS. Proc Natl Acad Sci USA 113: E8316-E8325, 2016.

36. Martín MC, Balfagón G, Minoves N and Blanco J: Androgen deprivation increases neuronal nitric oxide metabolism and its vasodilator effect in rat mesenteric arteries. Nitric Oxide 12: $163-176,2005$

37. Raivich G, Bohatschek M, Da Costa C, Iwata O, Galiano M, Hristova M, Nateri AS, Makwana M, Riera-Sans L, Wolfer DP, et al: The AP-1 transcription factor c-jun is required for efficient axonal regeneration. Neuron 43: 57-67, 2004.

38. Giguère V, Yang N, Segui $P$ and Evans RM: Identification of a new class of steroid hormone receptors. Nature 331: 91-94, 1988.

39. Hong H, Yang L and Stallcup: MR Hormone-independent transcriptional activation and coactivator binding by novel orphan nuclear receptor ERR3. J Biol Chem, 274: 22618-22626, 1999.

40. Zhang Z and Teng CT: Interplay between estrogen-related receptor alpha (ERRalpha) and gamma (ERRgamma) on the regulation of ERRalpha gene expression. Mol Cell Endocrinol 264: 128-141, 2006.

41. Sladek R, Bader JA and Giguère V: The orphan nuclear receptor estrogenrelated receptor alpha is a transcriptional regulator of the human medium-chain acyl coenzyme A dehydrogenase gene. Mol Cell Biol 17: 5400-5409, 1997.

42. Cao J and Patisaul HB: Sexually dimorphic expression of hypothalamic estrogen receptors $\alpha$ and $\beta$ and Kiss1 in neonatal male and female rats. J Comp Neurol 519: 2954-2977, 2011.

This work is licensed under a Creative Commons Attribution-NonCommercial-NoDerivatives 4.0 International (CC BY-NC-ND 4.0) License. 